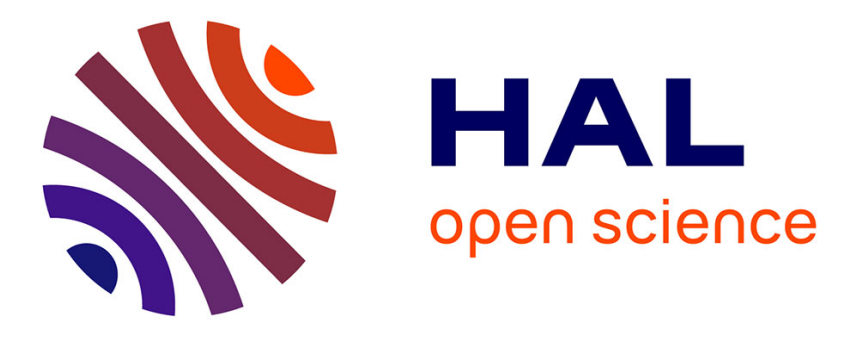

\title{
Intégrer une " éthique préventive " dans le processus de relèvement post-catastrophe : résilience, adaptation et " reconstruction préventive "
}

Annabelle Moatty, Freddy Vinet, Stéphanie Defossez, Jean-Philippe Cherel, Frédéric Grelot

\section{To cite this version:}

Annabelle Moatty, Freddy Vinet, Stéphanie Defossez, Jean-Philippe Cherel, Frédéric Grelot. Intégrer une " éthique préventive " dans le processus de relèvement post-catastrophe : résilience, adaptation et " reconstruction préventive ". La Houille Blanche - Revue internationale de l'eau, 2018, 5-6, pp.1119. $10.1051 / \mathrm{lhb} / 2018046$. hal-02518278

\section{HAL Id: hal-02518278 \\ https://hal.science/hal-02518278}

Submitted on 28 Apr 2020

HAL is a multi-disciplinary open access archive for the deposit and dissemination of scientific research documents, whether they are published or not. The documents may come from teaching and research institutions in France or abroad, or from public or private research centers.
L'archive ouverte pluridisciplinaire HAL, est destinée au dépôt et à la diffusion de documents scientifiques de niveau recherche, publiés ou non, émanant des établissements d'enseignement et de recherche français ou étrangers, des laboratoires publics ou privés.

\section{(c)(1)}

Distributed under a Creative Commons Attribution| 4.0 International License 


\title{
Intégrer une « éthique préventive » dans le processus de relèvement post-catastrophe : résilience, adaptation et « reconstruction préventive »
}

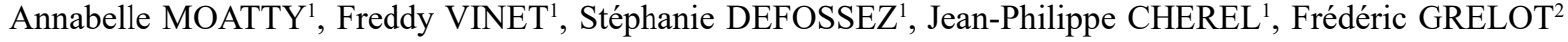 \\ 1. Unité Mixte de Recherche Gouvernance Risques Environnement et Développement (GRED), Université Paul Valéry / Institut de Recherche \\ pour le Développement (IRD), 2 rue du Pr Henri Serres, 34000 Montpellier - amoatty@yahoo.fr-freddy.vinet@univ-montp3.fr-stephanie. \\ defossez@univ-montp3.fr-jean-philippe.cherel@univ-montp3.fr \\ Unité Mixte de Recherche Gestion de l'Eau, Acteurs, Usages (G-EAU), IRSTEA (Institut national de recherche en sciences et technologies \\ pour l'environnement et l'agriculture), 361 rue JF Breton, 34000 Montpellier - frederic.grelot@irstea.fr
}

\begin{abstract}
RÉSUMÉ. - La reconstruction, entendue comme l'ensemble des mesures pour rétablir un fonctionnement acceptable du territoire, est une notion de plus en plus traitée dans la littérature scientifique des risques. Elle peut faire l'objet, sans que cela soit systématique, de stratégies d'adaptation collectives et individuelles, planifiées et spontanées, aux objectifs différents. L'anticipation de la reconstruction est un enjeu majeur pour optimiser le potentiel préventif et pour fluidifier la prise de décision en période post-catastrophe. L'étude des phases du processus par le biais d'un retour d'expérience à moyen et long terme permet d'en dégager des règles et de définir des blocages et leviers d'action au regard des objectifs de la Réduction des Risques de Catastrophe et de l'Adaptation au Changement Climatique pour mettre en œuvre les principes du Développement Durable. Les méthodes d'enquêtes, l'analyse documentaire, et la spatialisation des résultats permettent la collecte des données et leur analyse plusieurs années après la catastrophe. Nous nous appuyons sur l'analyse de 2 reconstructions post-catastrophe : l'Aude (notamment Cuxac-d'Aude) et le Var, suite aux inondations de 1999 et 2010. Des exemples d'adaptations ont été intégrés à la reconstruction mais de manière opportuniste sans véritable stratégie globale. Il ressort que l'événement catastrophique à lui seul ne peut être le déclencheur d'une adaptation au risque et que la mise en œuvre d'une reconstruction préventive ne s'improvise pas : le travail d'anticipation est aussi nécessaire que nécessairement limité par le besoin d'ajustement ad hoc.
\end{abstract}

Mots-clés : reconstruction post-catastrophe, ethique préventive, adaptations, résilience

\section{Integrating a "preventive ethic" in the post-disaster recovery process: resilience, adaptations and "preventive reconstruction"}

\begin{abstract}
Post-disaster recovery, understood as the set of measures allowing to restore an acceptable functioning of the territory, is a concept which has appeared relatively recently in the scientific and geographical literature of risks. It can be, yet not automatically, addressed by collective and individual, planned and spontaneous coping strategies, which the objectives may vary according to the interests. The anticipation of reconstruction is a major challenge to optimize the integration of a "preventive ethic" and, more generally, to facilitate decision-making in the post-disaster period. The study of the phases of the process through medium- and long-term feedback allows us to identify rules, and to define constraining factors and pitfalls in the one hand, and favourable conditions and means of action in the other hand, regarding the Disaster Risk Reduction and Climate Change Adaptation to pursue Sustainable Development objectives. This method combines the social science surveys, documentary analysis and GIS, and is carried out several years after the disaster. To illustrate the reasoning and clarify the links between adaptations and resilience, we rely on the analysis of two postdisaster reconstructions in the French metropolitan area: the Aude department after the floods of 12 and 13 November 1999, and the Dracenie area (Var department) following the floods of 15 and 16 June 2010. They are also completed by an analysis of the evolution of risk and disaster management perceptions, and an analysis of long-term memory in the municipality of Cuxac-d'Aude. Examples of adaptations have been integrated into the reconstruction but opportunistically without a real overall strategy. It appears that the catastrophic event alone cannot be the trigger for an increase of resilience and a real strategy of adaptation to risk. Henceforth, the implementation of a preventive reconstruction cannot be improvised: the anticipation effort is as necessary as necessarily limited by the need for ad hoc adjustment of recovery strategies.
\end{abstract}

Key-words: post-disaster recovery, preventive ethic, adaptations, resilience

\section{INTRODUCTION}

La reconstruction est un processus spontané et planifié qui induit deux modalités : rebâtir à l'identique ou bien repenser les partis pris d'aménagement du territoire [Clinton, 2006 ; De Vanssay, 2010]. Par son inscription dans le temps long, la reconstruction se distingue des phases de post-crise et de réhabilitation qui sont limitées au rétablissement provisoire des fonctions vitales de la société et au relogement temporaire des sans-abris [Aysan et Davis, 1993 ; Oliver-Smith, 1991]. On a longtemps fait, en France et dans les pays développés, comme si les sommes d'argent drainées par le 
processus d'indemnisation et par la solidarité étaient suffisantes pour reconstruire efficacement. Pourtant, la récurrence des catastrophes, parfois sur les mêmes territoires, interroge l'efficacité des logiques préventives et curatives. Face à ce constat et au nom d'une rationalité économique, le concept de reconstruire mieux (traduction du Build Back Better $B B B$ ) fut développé à l'échelle internationale et érigé en injonction dans le Cadre d'Action de Sendai pour la période 2015 - 2030 [UNISDR, 2015]. La reconstruction peut être saisie comme une "fenêtre d'opportunité » [Christoplos, 2006] pour réduire les vulnérabilités et améliorer la résilience des sociétés et territoires. Elle peut faire l'objet, sans que cela soit systématique, de stratégies d'adaptation collectives et individuelles. L'étude du processus par le biais d'un retour d'expérience (RETEX) à moyen et long terme permet d'en dégager des règles, et de définir des facteurs contraignants et conditions favorables au regard des objectifs de prévention et de durabilité [Moatty, 2015]. Cette méthode qui combine les enquêtes (entretiens et questionnaires), l'analyse documentaire, et la spatialisation des résultats, est menée plusieurs années après la survenue de la catastrophe pour être en mesure d'analyser les conséquences des processus qui s'étendent sur des décennies [Oliver-Smith, 1991 ; Gaillard, 2002 ; Moatty et al., 2017].

La réflexion proposée s'appuie sur les résultats du projet de recherche RETINA (Résilience des territoires face à l'inondation : pour une approche préventive par l'adaptation post-évènement, financé par le programme Risque Décision et Territoire du Ministère de la Transition Ecologique et Solidaire $)^{1}$. Il se fixe pour objectif la qualification des possibilités d'adaptation des systèmes en période post-catastrophe (réparation, reconstruction, réorganisation). Trois questionnements structurent l'analyse : la phase post-catastrophe est-elle un temps privilégié pour la conception et mise en œuvre de stratégies d'adaptation ?, comment caractériser les liens entre résilience et adaptation dans le contexte des périodes de relèvement ?, à quoi cherche-t-on à s'adapter et quels sont les obstacles et leviers d'action à l'élaboration d'une stratégie de reconstruction préventive ? Ces questionnements sont illustrés par l'analyse de deux études de cas : la reconstruction dans le département de l'Aude après les inondations des 12 et 13 novembre 1999, et celle de la Dracénie suite aux inondations des 15 et 16 juin 2010 dans le département du Var, complétés par l'analyse de l'évolution des perceptions du risque et de sa gestion ainsi que celle de la mémoire à Cuxac-d'Aude.

\section{CADRAGE SCIENTIFIQUE ET CONCEPTUEL}

\section{II.1. La reconstruction comme opportunité préventive - reconstruire mieux, adaptations et résilience}

La reconstruction post-catastrophe mobilise des ressources et des énergies considérables. A ce titre, elle suscite parfois des conflits et induit des réactions face au risque qui ne vont pas toujours dans le sens d'une prévention durable. Nous entendons par là, une prévention qui s'organise dès la phase de reconstruction et qui garde la trace de l'évènement catastrophique [Vinet, 2010]. Il s'agit de reconstruire mieux en réduisant le risque à l'origine de la catastrophe (donc en agissant sur l'aléa et sur les causes profondes des vulnérabilités) sans

1. https://irsteadoc.irstea.fr/oa/PUB00057822-resilience-des-territoiresface-inondation-pour-un.html amplifier ou générer d'autres risques. L'injonction au $B B B$ ne plaide pas en faveur d'une attitude qui consisterait à attendre la catastrophe pour mettre en œuvre une politique de prévention mais de tirer profit des conditions de la période post-catastrophe afin de mettre en place des mesures préventives qui peinent à l'être en temps normal. En effet, certaines mesures demandent un investissement collectif jugé inacceptable par l'opinion publique au regard du caractère virtuel du risque en dehors des périodes de post-crise. La prise de conscience des vulnérabilités générée par la crise fait accepter les investissements préventifs [Anderson et Woodrow, 1989 ; Bourrelier et al., 1997]. Cependant, plusieurs obstacles se dressent de manière systématique devant la volonté des acteurs de reconstruire différemment : 1) la «compression temporelle » [Olshansky et al., 2012] qui évoque, dans les premiers mois après une catastrophe, le besoin de rapidité de l'arbitrage et l'action dans un contexte d'incertitudes, 2) l'inadéquation des procédures existantes qui ne permettent pas de gérer ce besoin de rapidité ni la nécessité d'inscrire les stratégies conçues pendant la reconstruction dans le temps long des politiques d'aménagement et de prévention. Face à ces obstacles, les acteurs du processus mettent en place une gouvernance ad hoc pour faciliter et accélérer les prises de décisions en opérant un double décloisonnement : à la fois sectoriel en réunissant les acteurs de l'aménagement du territoire, de la gestion des risques, de l'économie et de l'action sociale, et géographique en outrepassant les limites administratives [Moatty et al., 2017].

Au travers de l'étude menée dans le cadre du projet RETINA, nous avons cherché à comprendre comment les adaptations individuelles et collectives contribuent à la résilience des sociétés et territoires à différentes échelles. Nous partons donc du postulat que la résilience (à la fois entendue comme état et comme processus) est influencée par les mesures d'adaptation qui sont mises en œuvre de manière spontanées ou planifiées à différentes échelles territoriales et temporelles. La résilience est un phénomène complexe et dynamique qui renvoie à la triple capacité des systèmes : la résistance pendant la crise, la capacité de récupérer et de rebondir immédiatement après le choc et celle de s'adapter et se transformer dans le temps long [Reghezza et al., 2012 ; Quenault, 2014 ; Reghezza et Rufat, 2015]. La résilience s'analyse donc dans le cadre des théories sur le fonctionnement des systèmes complexes et à ce titre, la somme des adaptations ne fait pas à elle seule la résilience de la société soumise à l'effort de reconstruction : les deux notions entretiennent des relations complexes. La notion d'adaptation suppose un changement alors que la résilience, au moins au début du processus de sortie de crise et de reconstruction, suppose de résister donc de maintenir une stabilité dans le fonctionnement et l'état du système [Reghezza et Rufat, 2015]. Le développement des concepts d'adaptation et de $B B B$ est contemporain de l'avènement de celui de résilience [Djament-Tran et al., 2012 ; Béraud, 2013]. La question sous-jacente est d'identifier les types de réponses mises en œuvre à des échelles de temps variables qui permettent de gérer la crise et de récupérer, c'est-à-dire d'être résilient. L'analyse de ces réponses se fait par le prisme d'ajustements composés d'une série de transformations permettant de s'adapter. Pour mener à bien cette analyse, nous avons adapté la méthode de RETEX à l'analyse du processus de reconstruction depuis la sortie de crise jusqu'au développement à moyen et long terme.

\section{II.2. Une analyse rétrospective à moyen et long terme}

Le RETEX est un mode d'évaluation réalisé de manière quasi systématique après des inondations, il est diligenté 
par le ministère en charge de la prévention des risques. Ces RETEX, pluridisciplinaires et multisectoriels, sont menés par des inspecteurs des différents ministères ou corps de l'Etat. Ils sont également pratiqués en interne par d'autres ministères (Intérieur, Défense, Finances) ou par des acteurs de la gestion de crise et de la prévention dans le silo de leur secteur de compétence. En France, les RETEX les plus diffusés sont issus de missions d'inspections interministérielles et de missions parlementaires [Mariani, 1994 ; Galley et Fleury, 2001 ; Lefrou et al., 2000 ; Ledoux, 2000 ; Lefort et Koulinski, 2011 ; Collombat, 2012], et sont rendus publics dans l'année qui suit le sinistre. L'exercice peut s'assimiler à une démarche de débriefing [Wybo et al., 2003] pour déterminer les causes d'un évènement dommageable et en retirer des enseignements en matière de réduction des risques. Parmi les autres objectifs, on compte le recensement des dommages, le retour réflexif sur les procédures employées et le bilan des actions. La recherche des responsabilités n'est pas clairement affichée car politiquement sensible même si elle transparaît parfois et certains RETEX donnent lieu à des anicroches entre les acteurs locaux. Ils s'appuient sur les chiffrages de dommages provisoires destinés à cadrer à la hâte l'enveloppe financière nécessaire à la remise en état et à la reconstruction. C'est ainsi qu'après les inondations des 12 et 13 novembre 1999, le Premier Ministre vint en visite dans l'Aude dès le 18 novembre annonçant une enveloppe de 600 millions de francs (environ 90 millions d'euros) pour la reconstruction. Toutefois, les RETEX présentent des insuffisances dans l'analyse des phases de post-crise et de reconstruction [Hubert et Ledoux, 1999 ; Moatty, 2015], et ce malgré les recommandations en ce sens de plusieurs grands corps de l'Etat.

La loi du 13 juillet 1982 est souvent invoquée lorsqu'il s'agit d'expliquer le manque d'investissement dans l'adaptation post-événement. Par l'indemnisation des victimes de catastrophes naturelles, elle garantit un remboursement des dommages qui, nonobstant quelques contentieux, satisfait une grande majorité de sinistrés comme l'ont montré nos enquêtes dans l'Aude. De fait, tout se passe comme si la réparation post-catastrophe était un dû, un pansement que la collectivité nationale se devait d'assurer aux sinistrés afin d'apaiser la douleur (réelle) et parfois la colère de ces derniers. Cependant, Douard [2004] signale que " la réparation est peu administrée en France ». Sa gestion est confiée en partie aux assureurs qui ont en échange un marché captif (extension de garantie obligatoire) et une garantie stop loss quasi illimitée de l'Etat par le biais de la Caisse Centrale de Réassurance. Ce système est l'un des plus solidaires au monde et les enquêtes auprès des sinistrés révèlent des taux de satisfaction de l'ordre de 80 à $90 \%$ pour les remboursements des biens assurables. Ce système est considéré comme coûteux ce qui est discutable puisque le coût moyen de la garantie Cat Nat est de l'ordre de 20 à 40 euros par an et par foyer. Ce coût peut être considéré comme absorbable par nos sociétés développées. En revanche, le système est inefficace en termes de prévention car il ne contribue pas à la réduction des risques sur le long terme. L'assurance est une compensation des dommages et non une réparation préventive. De nombreux rapports et RETEX post-inondation ont souligné son effet déresponsabilisant quant à la mise en œuvre de mesures de prévention [Bourrelier et al., 1997 ; Fleury, 2001]. Force est de constater que même en monnaie courante et à enjeux constants, le coût des dommages liés aux inondations augmente [Bourguignon, 2013].

\section{MÉTHODOLOGIE DU RETEX À MOYEN ET LONG TERME}

\section{III.1. La collecte de données pour l'analyse des adaptations collectives}

Nous avons utilisé la méthodologie des RETEX pour collecter les informations et expériences afin de les capitaliser. Cette méthode, telle que définie dans les documents ministériels [Huet, 2005], a été adaptée aux spécificités de cette étude : une analyse sur le temps moyen et long qui dépasse la méthode à dires d'experts. Pratiqué sur le temps long, le RETEX est un outil de suivi de la reconstruction et de progrès itératif pour les stratégies de gestion. Les objectifs peuvent être regroupés en quatre grands ensembles [Moatty, 2015]. Le premier vise la mise à jour des données sur le bilan de la reconstruction (et sur celui de la catastrophe dans une moindre mesure). Dans cet objectif, une synthèse thématique a été réalisée pour constituer un état des lieux des connaissances afin d'élaborer les grilles d'entretien et de les adapter aux personnes soumises à l'enquête (PSE). Il s'agit donc d'entretiens ciblés fondés sur les constats faits lors des RETEX précédents d'une part, et sur le rôle de la personne interrogée d'autre part. Dans ce cas précis le but est d'analyser rétrospectivement le processus pour retracer la trajectoire de reconstruction. L'effort de contextualisation a été particulièrement important afin de répondre au deuxième objectif : analyser la stratégie globale de reconstruction afin d'en extraire les blocages et les leviers d'action. La représentation cartographique permet de spatialiser les résultats et ainsi d'analyser leurs interactions de manière globale. Vient ensuite l'objectif d'analyse de l'efficacité des mesures d'adaptations préventives qui est autorisée par le recul temporel (qui doit cependant être compensé par le croisement des sources de données). Enfin, le dernier objectif est de dégager des règles générales en matière de reconstruction préventive.

La première étape du travail a consisté en une phase de recherche et synthèse bibliographique sur la reconstruction et sur les processus d'adaptation sur les deux territoires de l'Aude et du Var. Ceci nous a permis de sélectionner les communes sur lesquelles une analyse plus poussée allait être menée afin de conduire la phase d'entretiens exploratoires auprès des personnes ressources qui étaient en poste lors de la gestion de ces évènements. La phase d'enquêtes (entretiens semi-directifs) s'est étendue sur une année, de janvier à décembre 2014 pour un total de 51 PSE (28 dans l'Aude et 23 dans le Var). Certaines personnes ont été interrogées plusieurs fois et le nombre d'entretiens s'élève à 58 (32 dans l'Aude et 26 dans le Var). Les entretiens ont été complétés par des journées d'observation avec les acteurs du milieu associatif et avec les syndicats de bassin (Syndicat Intercommunal d'Aménagement de la Nartuby dans le Var et Syndicat Mixte des Milieux Aquatiques et des Rivières dans l'Aude). En parallèle nous avons analysé 170 documents mis à disposition par les PSE pour compléter et valider les informations issues des entretiens d'une part, et pour mettre à jour les bilans issus des RETEX réalisés « à chaud », juste après les évènements [Lefrou et al., 2000 ; Ledoux, 2000 ; Cour des Comptes, 2012 ; Rouzeau et al., 2010 ; Collombat, 2012].

\section{III.2. L'analyse des adaptations individuelles}

L'endommagement des particuliers pèse lourd dans le bilan des catastrophes. Cette entrée par l'individu présente l'intérêt d'estimer la part d'investissement personnel dans la gestion des risques et particulièrement suite à un évènement 
grave. La pertinence de l'analyse s'accentue grâce une approche diachronique sur le moyen terme. L'évènement déclenche-t-il une démarche de reconstruction préventive? Plusieurs années après la catastrophe, les adaptations, si elles existent, restent-elles d'actualité ?

Pour répondre à ces questionnements les enquêtes auprès des populations donnent des éléments de réponse. A travers l'exemple des basses plaines de l'Aude touchées par des inondations en novembre 1999, des études de perception tentent d'analyser des évolutions à moyen terme. En 2004 une étude de perception a été menée sur deux communes (Coursan et Cuxac-d'Aude), quelques années après la crue de 1999 [Defossez, 2009]. Il s'agissait d'étudier leur perception de l'évènement mais aussi plus globalement, de la gestion des risques. Dans une perspective de RETEX sur le moyen terme, les mêmes questionnaires ont été renouvelés dix ans après en 2015. La volonté d'interroger les mêmes domiciliations est fondée sur le postulat que l'évènement passé influence les initiatives à court terme. De plus, les adaptations individuelles restent ponctuelles et les PSE formulent une attente d'adaptations et protections collectives de l'échelle institutionnelle. En 2004, 275 questionnaires ont été réalisés (avec un taux de retour de $50 \%$, soit 143 questionnaires).

Cette approche diachronique est articulée autour de questionnements sur l'identification et la détermination des mesures d'adaptations, ainsi que sur l'élément déclencheur à l'échelle des foyers. Le moyen terme (2004) et long terme (2015) donnent l'opportunité d'analyser les évolutions telles que la poursuite ou l'arrêt des mesures d'adaptations individuelles par exemple. Les enquêtes apportent des éléments d'analyse sur les relations entre échelle individuelle et dimension collective de la gestion des risques.

\section{RÉSULTATS}

\section{IV.1. Apport des RETEX à moyen et long terme pour retracer les trajectoires de reconstruction}

La reconstruction post-catastrophe n'est pas un processus neutre, les stratégies mises en œuvre contribuent à favoriser certains secteurs de l'économie, de la société et des territoires, répondant ainsi à une logique qui dépasse le cadre de la gestion des risques. Dans ce contexte, le RETEX permet d'identifier les méthodes et prérequis qui ont eu un impact positif et/ou négatif sur la gestion de l'évènement, d'évaluer l'efficacité des mesures prises et "d'accéder à la capitalisation d'expériences » [Leone, 2007]. Les RETEX prennent plusieurs formes selon les buts poursuivis, de l'enquête judiciaire aux RETEX institutionnels en passant par les programmes de recherche. Le RETEX à moyen et long terme est peu développé et l'on dispose de très peu d'exemples dans la littérature française. L'objectif est de mesurer les effets indirects d'un évènement sur les trajectoires de développement. Il se distingue donc des RETEX classiques par la période à laquelle il est réalisé (plusieurs années après la catastrophe) [Moatty et al., 2017]. La première étape est celle de la synthèse thématique des travaux réalisés sur la reconstruction et sur les logiques d'adaptation qui sont impulsées ou confirmées en phase post-catastrophe.

Pour la Dracénie (Var), nous avons réalisé un RETEX à moyen terme (été 2014) au cours duquel la reconstruction était encore en cours. Pour l'Aude il s'agit d'un RETEX long terme puisqu'il a été réalisé 15 ans après la survenue de la catastrophe, la reconstruction était achevée en grande majorité. Dans les deux cas, il est nécessaire de pallier la distance temporelle qui éloigne les PSE de l'objet d'étude. Il s'agit d'opérer une validation (croisement des données) et une réanalyse des données brutes, complétées par l'étude de la bibliographie existante ainsi qu'avec l'étude des documents d'époque (arrêtés ministériels, préfectoraux et municipaux, courriers, revue de presse, documents techniques, documents de travail, rapports, notes, comptes rendus de réunions, etc.). Cette procédure permet de compléter la méthode dite à dires d'experts. Une partie des données a été analysée d'un point de vue qualitatif afin de produire une description factuelle de la phase de reconstruction et ainsi fournir des explications des trajectoires de reconstruction et de développement. Les données quantitatives viennent appuyer le raisonnement et illustrer les théories exposées.

\section{IV.2. Caractéristiques des adaptations collectives - l'exemple emblématique de Durban-Corbières}

L'exemple de Durban-Corbières montre les efforts d'une municipalité pour saisir l'opportunité de reconstruire autrement et en particulier pour soustraire un maximum d'enjeux à la zone inondable. Avant les crues de 1999, l'urbanisation du village s'était étendue en direction des berges de la Berre (cf. figure 1). L'installation des populations et des activités dans cette zone à risque a été en partie permise par la période de calme hydrométéorologique qu'a connu le sud de la France entre les années 1970 et 1990. « Les crues de novembre 1999 ont "remis les pendules à l'heure" pour reprendre l'expression d'un élu de la commune » [Vinet, 2010].

La crue de 1999 qui a débité au maximum 1000 à $1100 \mathrm{~m}^{3} / \mathrm{s}$ dans la commune de Durban-Corbières [CETE, 2000] a rendu impraticables les ponts qui relient les deux parties du village. Les biens publics ont été la principale catégorie d'enjeux sinistrés. En ce qui concerne les biens privés, $87 \%$ des artisans et 100 foyers ont été sinistrés. La décision a été prise de reconstruire différemment en recomposant le territoire de manière à ce que les enjeux soient moins exposés et / ou vulnérables (cf. figure 2). Dès 2000, la zone du lotissement de l'Estrade a été convertie en zone d'expansion de crue. Les obstacles au réaménagement du territoire ont été nombreux, d'ordre foncier tout d'abord. Le temps nécessaire à la constitution d'un nouveau lotissement n'a pas permis de proposer aux sinistrés un terrain ou un logement immédiatement et la plupart des personnes dont le bien devait être délocalisé se sont relogées dans des communes voisines. Le nouveau lotissement ne fut ouvert qu'en 2003 après avoir été retardé par des propriétaires fonciers récalcitrants (opposition politique, spéculation foncière). Le centre de secours et la gendarmerie ont été délocalisés sur les terrains de l'ancienne cave coopérative aujourd'hui démolie après désaffectation. L'école maternelle, le trésor public et le camping ont également été reconstruits hors zones inondables (cf. figure 2). Cette redistribution des services et de l'habitat remet en cause la cohérence du village acquise par les aménagements autour de la Berre [Vinet, 2003]. Des travaux de protection ont aussi été effectués sur les ouvrages d'art. Dans le cas du pont Général Raffin, les remblais d'accès ont été abaissés de façon à laisser écouler les grandes crues sans constituer un obstacle infranchissable ni favoriser un phénomène embâcle-débâcle. Sur les quais de la Berre, les maisons ont été sinistrées mais aucune mesure de délocalisation, réduction des risques, ou d'adaptation à ce dernier n'a été prise du fait des surcoûts considérables liés à l'ancienneté des constructions et à leur configuration d'habitat groupé mitoyen.

Le cas de Durban-Corbières permet d'illustrer une reconstruction adaptative même si ce fut dans la douleur. La 


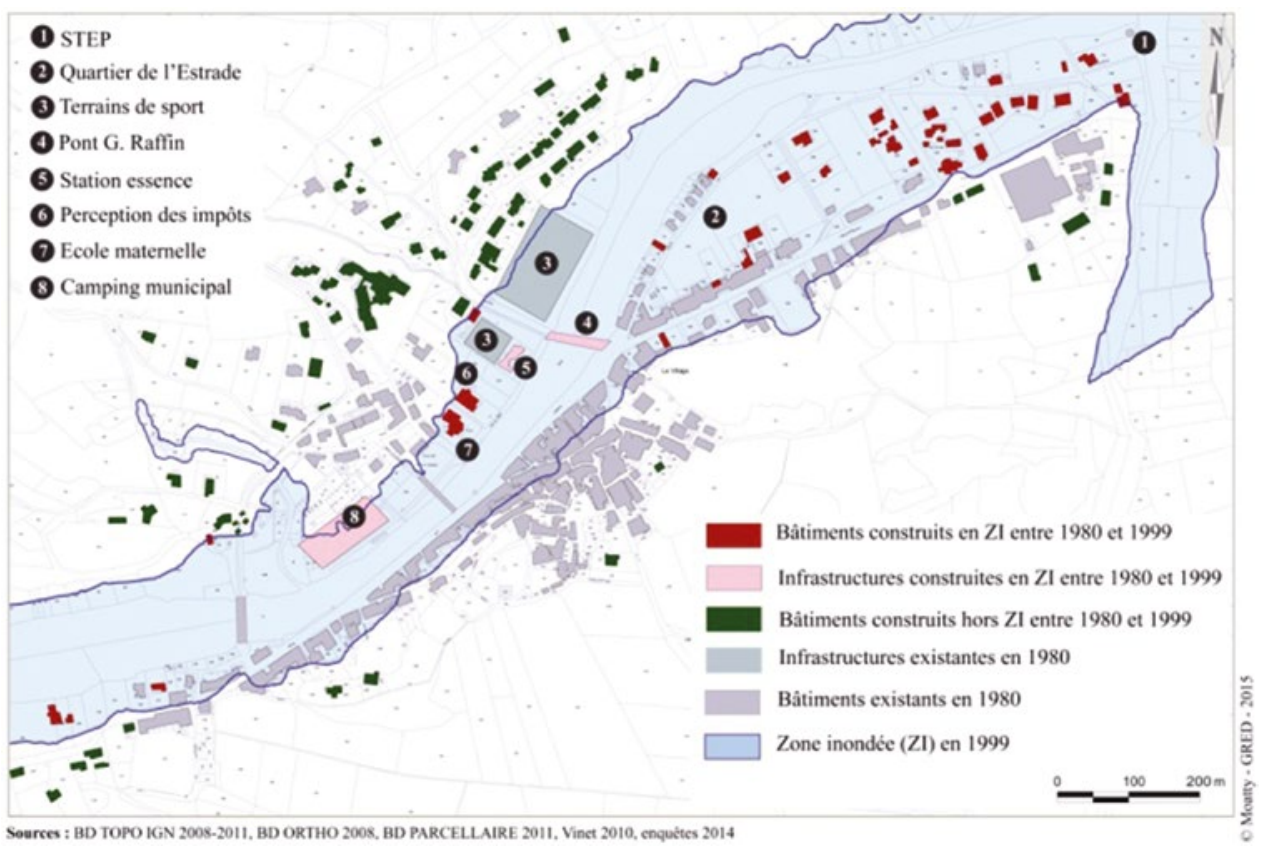

Figure 1 : Durban-Corbières avant les inondations de novembre 1999 [Moatty, 2015].

Légende: Cette carte représente la commune de Durban-Corbières, traversée par la Berre, dans le département de l'Aude, avant les inondations des 12 et 13 Novembre 1999. Les bâtiments y sont représentés ainsi que l'enveloppe de la zone inondée en 1999, zonage qui deviendra celui du PPRI de la commune. Les polygones dans les tons rouges sont les constructions implantées à l'intérieur de la zone inondable entre 1980 et 1999 : en bordeaux ce sont les bâtiments (quartier de l'Estrade) et en rosé ce sont les infrastructures construites à la même période (pont G. Raffin). Dans les tons verts, ce sont les bâtiments et infrastructures construits en dehors de la zone inondable pour la même période. Enfin en gris, ce sont les constructions existantes en 1980. Une partie du développement de la commune s'est faite vers les berges de la Berre.

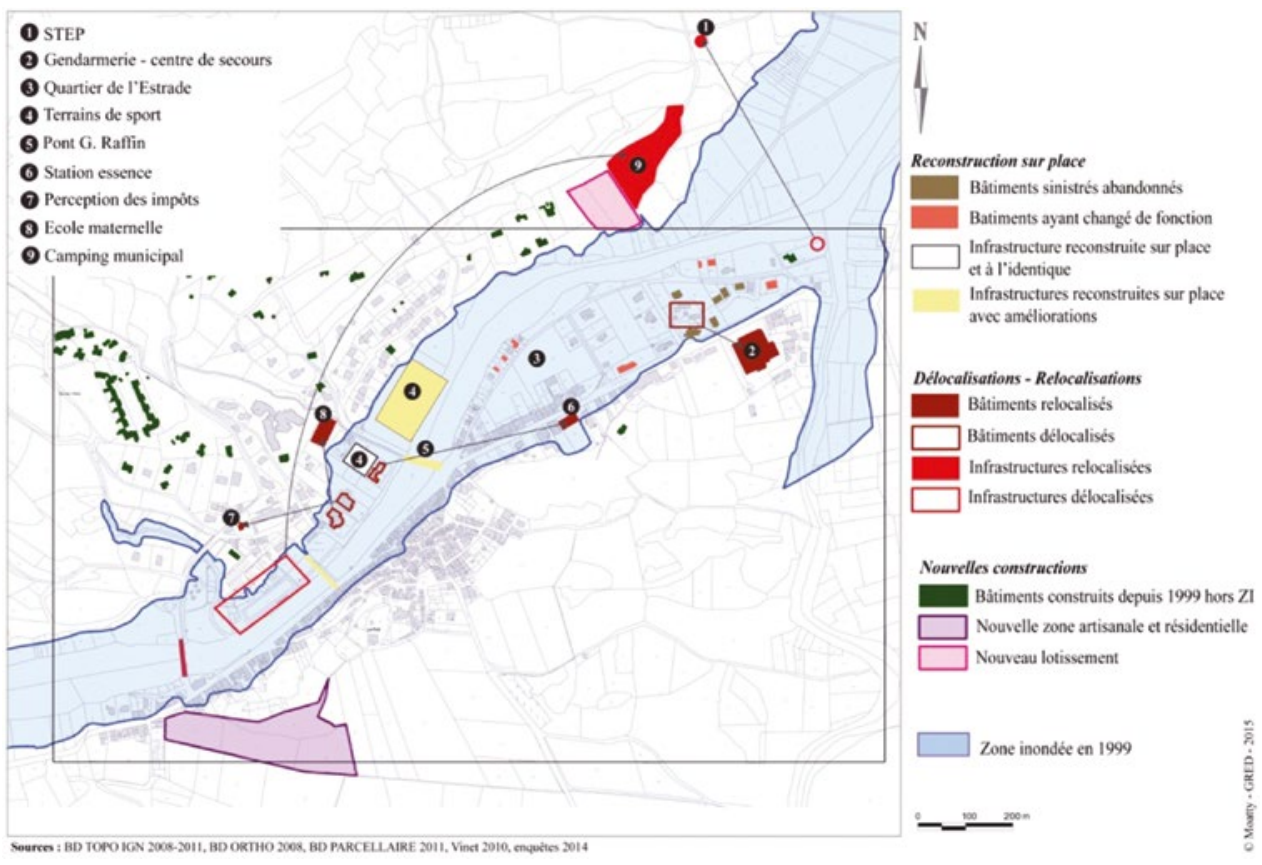

Figure 2 : Reconstruction et développement de Durban-Corbières suite aux inondations de 1999 [Moatty, 2015].

Légende: Cette carte représente la recomposition territoriale de la commune de Durban-Corbières après les inondations de novembre 1999. Le premier bloc d'informations concerne les reconstructions sur place et regroupe les bâtiments et infrastructures reconstruits sur place avec (polygones oranges et jaunes) et sans (polygones blancs et marrons) améliorations, préventives ou non. Le deuxième bloc représente les bâtiments et infrastructures qui ont été délocalisés et relocalisés ailleurs sur le territoire de la commune. Les flèches font le lien entre l'emplacement initial (polygones dans les tons de rouge et bordeaux pleins) et celui choisi en post-catastrophe (polygones avec contour uniquement). Enfin un troisième bloc concerne les constructions réalisées après 1999 (polygones roses et violets) ainsi que celles qui existaient en dehors de la zone inondable en 1999. Le redéveloppement de la commune s'est effectué dans l'objectif de réduire l'exposition des enjeux du territoire. 
recomposition du territoire intègre des mesures de réduction des risques qui ont été pensées en période post-catastrophe et qui n'auraient pas pu être réalisées sans la survenue de cette catastrophe de 1999 et des inondations suivantes en 2005 et 2006. Cependant, le dossier de la reconstruction à Durban-Corbières n'est toujours pas refermé puisqu'en 2016 il reste toujours deux habitations et des ateliers relais en bord de Berre qui n'ont pas été détruits. D'ailleurs les principaux acteurs de la reconstruction de la commune que nous avions rencontrés à plusieurs reprises entre 1999 et 2003 [Vinet, 2003] n'ont pas souhaité nous recevoir, écœurés par le temps et l'énergie passés à cette tâche, efforts sanctionnés par la récupération politique d'erreurs (inévitables) dans la conduite de certains projets.

\section{IV.3. Caractéristiques des adaptations individuelles}

Dans l'Aude, les adaptations individuelles concernent principalement la structure du bâti, jugée adaptée dès lors que la construction comporte un étage permettant aux habitants de trouver refuge. La moitié des populations interrogées à Coursan et Cuxac-d'Aude considèrent que leur habitation est adaptée au risque. Cependant, la construction d'une habitation à étage résulte de modes de vie spécifiques, qui dépassent le cadre de la réduction de vulnérabilité. Dans la commune la plus inondée (Cuxac-d'Aude), dans l'année qui a suivi la catastrophe, le nombre de mesures d'adaptation croit considérablement ( $1 / 3$ des PSE ont pris des mesures suite à 1999). L'analyse des permis de construire témoigne de mesures préventives pour faire face à un nouvel évènement, notamment avec la réalisation d'étage ou pièce refuge (plus de 30 demandes à Cuxac-d'Aude en 2000). Lors des premières enquêtes, il existait une volonté affichée de s'investir individuellement dans l'adaptation au risque. Cette dernière reposait majoritairement sur la mise en sécurité des personnes (pièce refuge) plus que sur la réduction de l'endommagement du bâti (revêtements hydrofuges, réseau électriques descendant, etc.).

Les réponses à l'enquête montrent que plus des trois quarts des PSE estiment que les institutions et notamment l'Etat doivent assurer la protection des riverains. Cependant, entre les deux dates d'enquête, la proportion des particuliers estimant avoir un rôle à jouer dans la gestion est passée d'un quart seulement à environ la moitié, sans toutefois que ces volontés s'accompagnent d'actions concrètes. Depuis quinze ans, peu de particuliers se sont investis dans la prise de mesures préventives. Pour les autres, les raisons de l'absence d'adaptation reposent principalement sur la méconnaissance du type de mesures à mettre en place, mais également par la méconnaissance du risque (surtout pour les populations n'ayant jamais vécu d'évènement grave et pour les nouveaux arrivants). Les communes périurbaines comme Coursan et Cuxac-d'Aude, proche de Narbonne et Béziers restent attractives et il existe un important renouvellement des populations (un tiers des interrogés par exemple ne résidait pas dans ce logement lors des premières enquêtes en 2004). L'information non systématique sur les risques, combinée à la sous-estimation d'un phénomène méconnu par les nouveaux arrivants, n'ouvrent pas la voie des adaptations individuelles.

Le faible investissement préventif individuel résulte de facteurs financiers, de méconnaissances (notamment en matière de possibilités techniques) ou de désintérêt. Pour le territoire étudié, des actions collectives d'envergure laissent penser que l'action collective suffit et justifie l'absence d'adaptation individuelle. A Cuxac-d'Aude, un projet de digues de second rang a été réalisé. La commune s'étend sur deux entités, le bourg ancien situé sur les berges de l'Aude et les quartiers plus récents (construits à partir des années 1960-70). Des digues ceinturent chacune des entités créant un chenal artificiel pour les eaux de crue. Ces digues, inaugurées en janvier 2015, sont potentiellement submersibles et donc vouées à gagner du temps pour l'organisation des évacuations en cas de crise majeure. Cette réalité n'est pas perçue comme telle par une large proportion des populations interrogées qui les considèrent comme une véritable protection face à l'arrivée des eaux. Ainsi, les populations accordent une large confiance à ces digues, ce qui peut être interprété comme le fait que les mesures de prévention collectives n'encouragent pas l'adaptation individuelle.

\section{DISCUSSIONS}

\section{V.1. Retour réflexif sur la méthode - les difficultés liées au temps moyen et long en SHS}

La première difficulté dans la tenue d'un RETEX à long terme tient à la mémoire de l'évènement. Retrouver les personnes en poste en 1999 et les années qui ont suivi a représenté la première difficulté. Une majorité d'élus des communes enquêtées sont restés à la tête de leurs mairies (64\% de mandats reconduits dans l'Aude). En revanche les gestionnaires de la reconstruction dans l'Aude ne sont que $39 \%$ à avoir gardé le même poste (cf. figure 3). La temporalité du retour d'expérience ( 15 ans après la catastrophe) et le contexte de décentralisation et de refonte des services de l'Etat («Lois Defferre »1982, 1983 et 1999) ont nécessité un effort considérable pour retrouver les gestionnaires de la reconstruction. Le travail aux archives (celles du département et celles des services qui les ont mis à notre disposition) et la multiplication des entretiens avec l'acteur ressource du bassin versant - le Syndicat Mixte des Milieux Aquatiques et des Rivières - ont été déterminants pour dépasser ce blocage.

Du fait principalement de la plus grande proximité temporelle de l'étude et de son objet dans le Var, ces contraintes ont été beaucoup moins prégnantes. En effet, $67 \%$ des élus ont été reconduits et $69 \%$ des gestionnaires étaient au même poste qu'en 2010 (cf. figure 3). En revanche, et contrairement aux idées reçues, les PSE qu'il s'agisse des gestionnaires ou des populations n'ont aucun mal à se souvenir du contexte et des modalités de la reconstruction tant elles ont été les acteurs de leur relèvement. "À ce titre, et contrairement au préjugé le plus courant, la capacité d'action et le moral des sinistrés ne sont pas réduits à néant suite à un désastre » [Moatty et al., 2017, p. 184]. Cependant, on note une perte d'informations concernant les données chiffrées ou les détails de certaines procédures élaborées ad hoc pour la reconstruction et abandonnées après. Le croisement des données issues des entretiens, avec les données des documents d'époque reste nécessaire car la mémoire est sélective. En effet, certaines erreurs et incohérences ont été mises en lumière par le croisement des informations alors qu'elles n'apparaissaient pas dans les retranscriptions des entretiens.

La deuxième difficulté rencontrée est liée à l'accessibilité des données. En effet, lors des premiers entretiens de repérage nous avions constaté la complexité d'accéder aux données et aux fichiers de gestion et de suivi autrement qu'en prolongeant les entretiens par des sessions de consultation des documents. Certaines données ont été perdues, notamment en ce qui concerne les données antérieures à la catastrophe qui permettent de comprendre le contexte dans lequel elle s'est déroulée. Certaines ont été détruites pendant 


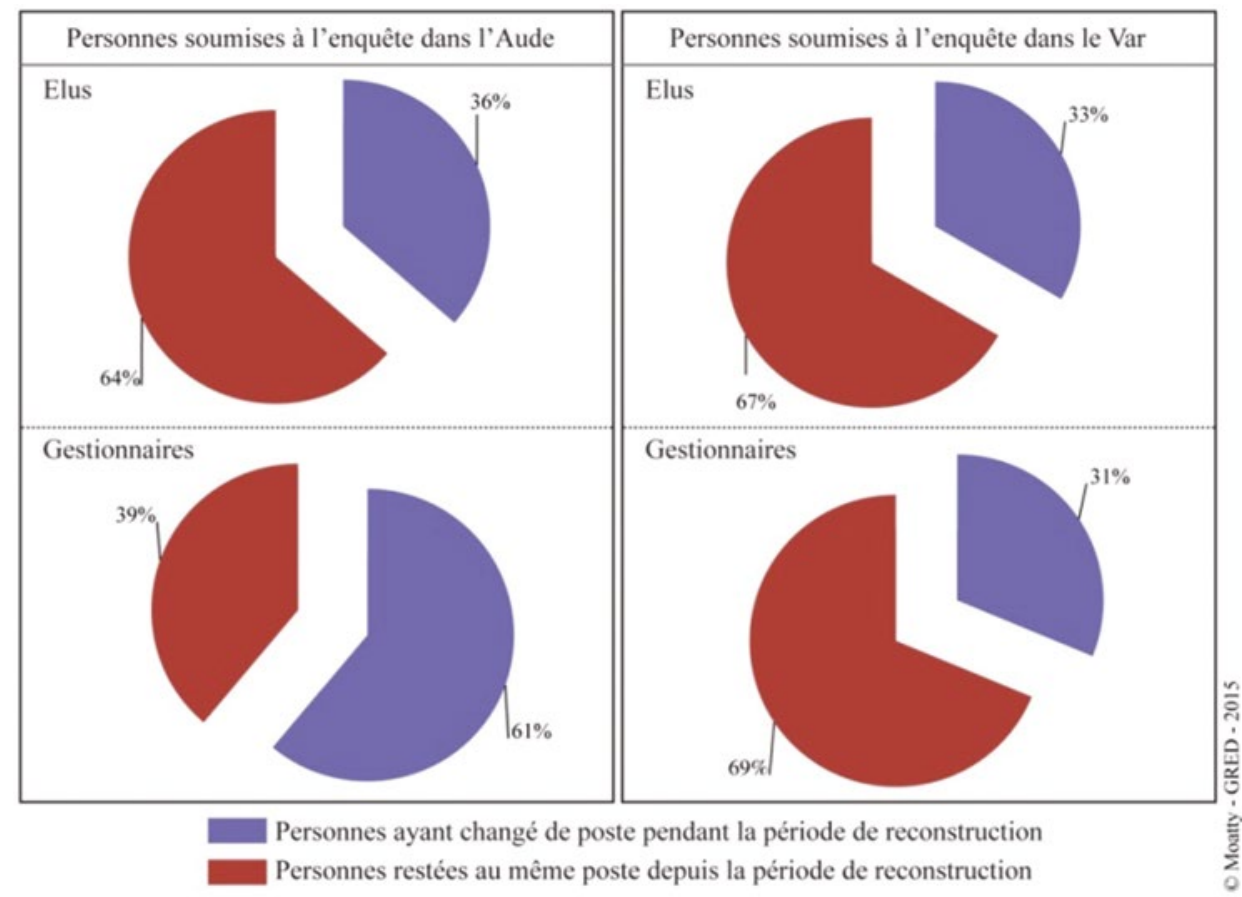

Figure 3 : Changement de postes des élus et gestionnaires de la reconstruction post-catastrophe [Moatty, 2015].

Légende: Ce graphique, réalisé à partir des entretiens réalisés dans l'Aude et le Var entre janvier et décembre 2014, représente la part de PSE (en bleu) qui a changé de poste depuis la date de la catastrophe (soit 1999 pour l'Aude et 2010 pour le Var), et celle (en rouge) qui est resté au même poste. Les deux cases du haut concernent les élus (maires des communes soumises à l'enquête) et celles du bas concernent les gestionnaires (institutions, associations, établissements publics de bassin versant, etc.).

la catastrophe et d'autres n'ont jamais été numérisées. La contrainte, évoquée plus haut, de la décentralisation et du remaniement des services de l'Etat a engendré quelques pertes d'informations. Les principaux documents ont néanmoins été conservés, parfois reconstitués et ont été mis à notre disposition, les documents manquants étant pour la plupart pourvoyeurs de détails complémentaires.

Pour les données concernant les inondations de 2010 en Dracénie, la problématique est différente, elle est liée au fait que les procédures soient encore en cours, les PSE ont préféré une consultation sur place en leur présence. Enfin, la troisième famille de difficultés est liée au manque d'information. Comme c'est le cas pour tous les travaux qui utilisent les méthodes d'enquêtes, nous avons expérimenté les problèmes de non-dit, de discours politique lissé. La durée des entretiens, comprise en $1 \mathrm{~h} 30$ et $5 \mathrm{~h} 30$, témoigne à la fois de la difficulté de collecter les informations voulues et du caractère chronophage de ce type de travail, mais aussi de la loquacité des PSE.

\section{V.2. Les mesures d'adaptation collectives et individuelles font-elles la résilience de la société et de son territoire ?}

Le manque d'anticipation, les nombreuses incertitudes qui pèsent sur les acteurs et l'urgence dans laquelle sont prises les décisions conduisent dans de nombreux cas à une persistance des risques et au creusement des écarts sociaux [Ingram et al., 2006 ; Gaillard, 2008 ; Moatty, 2015]. L'anticipation de la reconstruction est un enjeu majeur pour favoriser l'intégration d'une éthique préventive [Moatty et Vinet, 2016] et plus généralement, pour fluidifier la prise de décision en période post-catastrophe. L'éthique préventive peut être définie comme un " ensemble de règles qui assureraient une reconstruction durable sur le plan de la réduction des risques » [Vinet, 2010]. Le processus de reconstruction n'a pas pour point de départ un état zéro, il est soumis à des contraintes territoriales et politiques, et doit permettre d'apporter une réponse adaptée aux besoins des communautés sinistrées. Ce défi comporte également une dimension temporelle qui se traduit dans les premiers mois de la reconstruction par une " compression temporelle » [Olshansky et al., 2012] : les décisions et leurs mises en œuvre doivent être rapides et répondre à la fois aux besoins immédiats tout en prenant en compte ceux à moyen et long terme [Moatty, 2017].

Des sommes considérables sont investies dans la reconstruction des territoires et la grande majorité de ces fonds finance une reconstruction dite «à l'identique $»^{2}$. Le souci de l'Etat est d'encadrer l'utilisation des fonds publics et d'avoir la garantie qu'ils ne servent pas à pallier les manquements voire l'absence d'entretien des biens publics (dont la responsabilité incombe aux collectivités). Quand bien même certaines adaptations sont réalisées pour recomposer le territoire, elles ne sont pas nécessairement en phase avec les prérogatives de la prévention. Ainsi dans le Var, des bâtiments ont été construits dans des zones inondées en 2010. La raison invoquée était que les permis de construire avaient été attribués avant la catastrophe, pour autant aucune

2. L'espoir avait germé d'une évolution en ce sens puisque le projet de décret $n^{\circ}$ 2015-693 du 18 juin 2015 relatif à l'indemnisation des collectivités territoriales et de leurs groupements touchés par des événements climatiques ou géologiques était libellé « Dans le cas de travaux de réparation, le montant de la subvention prend en compte seules les dépenses nécessaires à la reconstruction du bien à fonctionnalités identiques, à l'exclusion de toute dépense d'extension ou d'amélioration du bien ». (Art. R. 1613-5). Dans le décret final on est revenu à la notion de « reconstruction à l'identique ». 
modification (incluant des mesures de réduction de la vulnérabilité) n'a été apportée aux permis. Le département de l'Aude a profité de la reconstruction pour mettre en œuvre une politique de remise à niveau des équipements et infrastructures. Cette démarche opportuniste fait figure de cas particulier qui ne tient pas seulement à l'occurrence des inondations de novembre 1999 mais à une situation politique locale favorable qui a été mise à profit après l'inondation. La mise à niveau des équipements publics n'avait pas pour objectif premier la réduction de la vulnérabilité même si elle a pu y contribuer en modernisant les biens vétustes ou en déplaçant parfois des équipements fortement exposés au risque. Les mesures structurelles lourdes, qui suscitent l'adhésion d'une grande partie de l'opinion publique, sont encore privilégiées au détriment d'actions de réduction des vulnérabilités - depuis l'échelle individuelle jusqu'à celle de la collectivité - fonctionnelles et organisationnelles, sur le moyen et long terme. En outre, ces mesures permettraient, si elles étaient dotées de moyens suffisants, de travailler sur les comportements à risque et sur les valeurs qui fondent des grands principes de la gestion des risques comme la solidarité amont-aval, la mutualisation des risques, etc.

Les dispositifs techniques et institutionnels de reconstruction sont installés empiriquement en temps voulu, mais rien ne garantit qu'ils soient systématiquement reconduits après un laps de temps assez long (10 ans ou 15 ans) ou lors d'une autre reconstruction. Il n'y a pas véritablement, quelle que soit l'échelle territoriale considérée, de capitalisation du savoir-faire en matière de reconstruction, ni d'organisation a priori. Les données sont conservées par les services instructeurs des dossiers ou bien déposées aux archives départementales (ce fut le cas pour l'Aude pour une partie des documents qui concernait les données agricoles et les biens publics) mais aucune analyse rétrospective n'est menée par les gestionnaires. La proximité géographique des départements peut jouer dans la transmission des connaissances en matière de reconstruction comme ce fut le cas lors des inondations du Gard en 2002. Des représentants de la mission reconstruction mise en place dans l'Aude ont été conviés à partager leur expérience opérationnelle avec les gestionnaires gardois qui ont repris la même structure de gouvernance (la cellule ou mission interministérielle de reconstruction) pour reconstruire leur territoire.

D'un point de vue pragmatique on peut considérer que les adaptations individuelles servent la résilience de la société. En effet, la construction de pièces refuges, qui représente l'essentiel des mesures d'adaptions individuelles, permet de réduire considérablement la vulnérabilité des occupants. Cependant les mesures restent trop anecdotiques pour qu'on puisse y voir une résilience de la société. Tout au plus les adaptations individuelles participent à la résilience individuelle. La méconnaissance des actions réduisant les dommages conduit à l'absence d'investissement personnel mais pas seulement. La phase post-crise est caractérisée par une forme d'urgence : retrouver un mode de vie acceptable qui entre en contradiction avec le temps nécessaire à la réalisation d'un projet d'adaptation de son habitation. Sur certains territoires, des diagnostics de vulnérabilité sont menés dans l'objectif final d'adapter les logements des particuliers. La démarche est intéressante - à condition d'être bien réalisée mais peu s'y intéressent et en bénéficient. On peut y voir un problème de responsabilisation des individus, qui en retour engendre un désengagement de leur part. Les institutions et gestionnaires ne laissent pas toujours la place aux citoyens dans les processus décisionnels et l'accompagnement pour la mise en place des mesures individuelles reste encore timide.

\section{CONCLUSIONS}

L'analyse des dispositifs législatifs et organisationnels, l'identification des blocages et des trajectoires de sortie de ces situations de blocage a permis de qualifier la pertinence de considérer la phase de reconstruction comme une opportunité préventive. La reconstruction, au moins dans les premiers mois après la catastrophe se doit d'être rapide et de permettre aux territoires de recouvrer rapidement des modalités de bon fonctionnement. Les questions de compétitivité économique et de développement pèsent lourd dans les prises de décisions. Et ce pour plusieurs raisons que l'on comprend d'ailleurs aisément : d'une part, la réponse aux attentes immédiates énoncées et induites par les citoyens, et d'autre part, le caractère supposé exceptionnel de l'évènement (l'événement comparable aux crues de juin 2010 dans le bassin de l'Argens datait de 1827). Les échéances du politique sont difficilement compatibles avec les temps longs des catastrophes et des recompositions des territoires. En ce sens on a un peu l'impression que dans le domaine de la prévention des risques comme dans d'autres ayant trait à l'organisation territoriale, les élus perdent la main et sont contraints par des phénomènes qu'ils ne maîtrisent pas faute de pouvoir se projeter dans les échelles de temps long.

Toutefois, il y a eu des exemples d'adaptation qui ont été intégrées à la reconstruction mais de manière opportuniste sans véritable stratégie globale. Il ressort que l'événement catastrophique à lui seul ne peut être le déclencheur d'une adaptation au risque. Il semble en particulier que la recomposition de la gestion de l'eau dans le département de l'Aude ait été un exemple de ce type d'opportunité non reproductible de façon systématique. La capitalisation locale des expériences, en tant que capacité d'adaptation est une clé de résilience. Elle passe par une centralisation des connaissances vernaculaires mais aussi par le décloisonnement et une habitude de travailler ensemble des services. Dans le cas de l'Aude cette habitude trouve ses racines dans la gestion de la reconstruction et a été perpétuée et entretenue par la restructuration de la gouvernance de l'eau à l'échelle du bassin versant. Reconstruire mieux ne s'improvise pas, cela suppose une sensibilisation et préparation des acteurs de la prévention, des gestionnaires publics aux citoyens en passant par les entrepreneurs et artisans. En mettant à l'épreuve le couple « bifurcation / continuité » dans les trajectoires de développement des sociétés et territoires, la période de reconstruction post-catastrophe questionne nos capacités d'anticipation, de préparation, de faire face et de faire avec.

\section{REMERCIEMENTS}

Cette recherche a été financée dans le cadre du programme Risques-Décision-Territoires « Résilience des Territoires face aux Risques » du Ministère de la Transition Ecologique et Solidaire. Ces résultats sont ceux du projet RETINA « résilience des territoires face à l'inondation : pour une approche préventive de l'adaptation post-événement ». Nous remercions également l'ensemble des personnes ayant accepté de donner un peu de leur temps pour répondre à nos questions.

Le Laboratoire de Géographie Physique de Meudon (UMR LGP, Paris 1 Panthéon Sorbonne / CNRS) est également remercié pour son soutien financier à la publication de cet article.

\section{RÉFÉRENCES}

Anderson M.B. Et Woodrow P. (1989) - Rising from the ashes: development strategies in times of disasters. Boulder and San Francisco, Westview Press/Unesco 
Aysan Y. Et Davis I. (1993) - Rehabilitation and reconstruction, Disaster Management Training Programme. Genève, United Nations Development Programme/Department of Humanitarian Affairs.

Bourguignon D. (2014) - Événements et territoires - le coût des inondations en France : analyses spatio-temporelles des dommages assurés, Université Paul Valéry - Montpellier III.

Bourrelier P.H. (Dir.) (1997) - La prévention des risques naturels : rapport de l'instance d'évaluation. La Documentation Française.

Beraud H. (2013) - Initier la résilience du service de gestion des déchets aux catastrophes naturelles : le cas des territoires urbains et de l'inondation. Université Paris-Est.

CETE (2000) - Bilan, pré-diagnostic et propositions pour l'aménagement de Durban après les inondations de novembre 1999.

Christoplos I. (2006) - The elusive "window of opportunity" for risk reduction in post-disaster recovery, Bangkok, ProVention Consortium.

Clinton W.J. (2006) - Key Propositions for Building Back Better. Lessons Learned from Tsunami. Recovery : Key Propositions for Building Back Better. New York, Office of the UN Secretary-General's, Special Envoy for Tsunami Recovery.

Collombat P.Y. (2012) — Se donner les moyens de ses ambitions : les leçons des inondations du Var et du sud est de la France. Rapport d'information, fait au nom de la Mission commune d'information Inondations dans le Var $\mathrm{n}^{\circ} 775$ (2011-2012) - 24 septembre 2012.

Cour Des Comptes. (2012) - Les enseignements des inondations de 2010 sur le littoral atlantique (Xynthia) et dans le Var 2999.

Defossez S. (2009) - Evaluation des mesures de gestion du risque inondation. Application au cas des basses plaines de l'Aude.

De VAnssay B. (2010) - La Reconstruction - Développement Durable et Réduction de la Vulnérabilité. Risques Infos Institut des Risques Majeurs, $\mathbf{n}^{\circ 24}$, 6-9.

Djament-Tran G., Le Blanc A., Lhomme S., Rufat S., ReghezzaZitT M. (2012) - Ce que la résilience n'est pas, ce qu'on veut lui faire dire. http://hal.archives-ouvertes.fr/hal-00679293/

DouARD P. (2004) - La politique de prévention des risques en France. Risques naturels et aménagement en Europe. Paris, Armand Colin, 14-17.

FLEURY A. (2001) - Cours d'agriculture urbaine, Versailles, France, ENSP.

GaILlard J.C. (2008) - Differentiated adjustment to the $1991 \mathrm{Mt}$ Pinatubo resettlement program among lowland ethnic groups of the Philippines. Australian Journal of Emergency Management, n' 23, 31-39.

GAILlARD J.C. (2002) - Implications territoriales et ethnoculturelles d'une crise volcanique : le cas de l'éruption du Mont Pinatubo aux Philippines. Annales de Géographie, nº27-628, 574-591.

Galley R. Et Fleury J. (2001) — Inondations : une mobilisation nécessaire, 24 propositions. Enregistré à la Présidence de l'Assemblée nationale le 14 novembre 2001. Commission d'enquête sur les causes des inondations répétitives ou exceptionnelles et sur les conséquences des intempéries afin d'établir les responsabilités, d'évaluer les coûts ainsi que la pertinence des outils de prévention, d'alerte et d'indemnisation, $n^{\circ} 3386$.

Hubert G. Et Ledoux B. (1999) — Le coût du risque... L'évaluation des impacts socioéconomiques des inondations. Presses de l'école nationale des Ponts et Chaussées, Paris.

Huet P. (2005) - La méthodologie des retours d'expérience après les accidents naturels. Première tentative de codification. Rapport n ${ }^{\circ}$ IGE/05/017, 85 p.

Ingram J. C., Franco G., Rumbaitis-Del Rio C., Khazai B. (2006) - Post-disaster recovery dilemmas : challenges in balancing short-term and long-term needs for vulnerability reduction. Environmental Science \& Policy, $\mathbf{n}^{\circ}$ 9, 607-613.

Ledoux B. (2000) - Retour d'expérience sur la gestion post-catastrophe dans les départements de l'Aude et du Tarn. Rapport pour le Ministère de l'Aménagement du Territoire et de l'Environnement (MEEM, ex-MATE), Bruno Ledoux Consultants, 70 p.
Lefort P. Et Koulinski V. (2011) - Crue du 15-16 juin 2010. Expertise post-crue. Tome 1, 2 et 3.

Lefrou C., Martin X., Labarthe J.P., Varret J., Maziere B., Tordjeman R., Feunteun R. (2000) - Les crues des 12, 13 et 14 novembre 1999 dans les départements de l'Aude, de l'Hérault, des Pyrénées Orientales et du Tarn. Rapport au Ministre de l'Aménagement du Territoire et de l'Environnement.

LeONE F. (2007) - Caractérisation des vulnérabilités aux catastrophes «naturelles »: contribution à une évaluation géographique multirisque (mouvements de terrain, séismes, tsunamis, éruptions volcaniques, cyclones). Mémoire d'Habilitation à Diriger des Recherches (HDR), Section 23 (Géographie), Université Paul Valéry - Montpellier III, Laboratoire GESTER.

Mariani T. (DIR.) (1994) — Rapport de la commission d'enquête sur les causes des inondations et les moyens d'y remédier. $\mathrm{N}^{\circ}$ 1641, Journal Officiel de 1'Assemblée Nationale du 4 novembre 1994.

MoAtтy A. (2017) — La reconstruction après les inondations : une opportunité préventive ? Connaissance et gestion des inondations. Tome 2, ISTE Ed.

Moatty A., Gaillard J.C., Vinet F. (2017) — Du désastre au développement : Les enjeux de la reconstruction post-catastrophe. Annales de géographie, $\mathbf{n}^{\circ}$ 714, 169-194.

Montty A. Et Vinet F. (2016) - Post-disaster recovery, the challenge of anticipation. E3S Web of Conferences 7, 17003, FLOODrisk 2016 - 3rd European Conference on Flood Risk Management.

Moatty A. (2015) - Pour une Géographie des Reconstructions post-catastrophe : Risques, Sociétés et Territoires.

Oliver-Smith A. (1991) - Successes and failures in post-disaster resettlement. Disasters, $\mathbf{n}^{\circ} \mathbf{1 5}, 12-23$.

Olshansky R.B., Hopkins L.D., Johnson L.A. (2012) — Disaster and recovery: processes compressed in time. Natural Hazards Review, $\mathbf{n}^{\circ} \mathbf{1 3}, 173-245$.

Quenault B. (2014) - La résurgence/convergence du triptyque catastrophe-résilience-adaptation pour (re)penser la « fabrique urbaine " face aux risques climatiques. Développement Durable \& Territoires (DDT), [En ligne], vol. 5, $\mathbf{n}^{\circ} 3$.

ReghezZa M. Et Rufat S. (2015) — Résiliences : sociétés et territoires face à l'incertitude, aux risques et aux catastrophes. ISTE éd.

Reghezza M., Rufat S., Djament G., Le Blanc A., Lhomme S. (2012) - What Resilience Is Not: Uses and Abuses. Cybergeo: European Journal of Geography; DOI 25554.

Rouzeau M., Martin X., Pauc J.C. (2010) - Retour d'expérience des inondations survenues dans le département du Var les 15 et 16 juin 2010. Ministère de l'écologie, de l'énergie, du développement durable et de la mer.

UNISDR (2015) - Cadre d'action de Sendai pour la réduction des risques de catastrophe 2015 - 2030. 18 Mars, Nations Unies, Bureau pour la Réduction des Risques de Catastrophe (UNISDR), A/CONF.224/CRP.1.

UNISDR (2005) - Hyogo Framework for Action 2005-2015. Building the Resilience of Nations and Communities to Disasters. World Conference on Disaster Reduction, 18-22 January 2005, Kobé, Hyogo, Japan.

VINET F. (2010) - Le risque inondation. Diagnostic et gestion. Editions Lavoisier Tec \& Doc collection SRD.

Vinet F. (2003) - Crues et inondations dans la France méditerranéenne. Les crues torrentielles des 12 et 13 novembre 1999 (Aude, Tarn, Pyrénées Orientales et Hérault). Editions du Temps, Nantes.

Wybo J.L., Godfrin V., Colardelle C., Guinet V., Remis Denis C. (2003) - Méthodologie de retour d'expérience des actions de gestion des risques. Programme « Evaluation et Prévention des Risques »- Convention MATE 07/2001 Ministère de l'Ecologie et du Développement Durable / Ecole des Mines de Paris. 